\title{
Cómo fomentar las competencias transversales en los estudios de Ciencias de la Salud: una propuesta de actividades
}

\section{New activities for developing generic skills in the health sciences}

Josep-E. Baños y Jorge Pérez.

Oficina de Coordinación y Evaluación Académica Facultad de Ciencias de la Salud y de la Vida

Universitat Pompeu Fabra (Barcelona)

\section{El proceso de convergencia europea de enseñanza superior incide en la necesidad de contemplar las competencias genéricas o transversales en la formación pregraduada. Dichas competencias son especialmente adecuadas para los futuros profesionales en ciencias de la salud. \\ El presente artículo pretende aportar algunos elementos sobre la bondad de su implementación así como sugerir diversas actividades para fomentarlas en el marco de los procesos docentes de las titulaciones. Las propuestas están contempladas a partir de experiencias positivas desarrolladas en la Facultad de Ciencias de la Salud y de la Vida de la Universidad Pompeu Fabra de Barcelona y en otros centros españoles.}

Palabras clave: Competencias genéricas. Aprendizaje activo. Métodos de enseñanza.

Correspondencia:

Dr. Josep-E. Baños

Departamento de Ciencias Experimentales y de la Salud.

Universitat Pompeu Fabra.

Dr. Aiguader, 80

08003-Barcelona

Teléfono: 34935422950

E-mail: jbanos@imim.es
The development of generic skills in undergraduate students is considered to be especially important in the process leading to the European Space of Higher Education, in the area of the health sciences in particular.

This paper explains the reasons for this importance and proposes a set of activities that may help to improve generic skills at undergraduate level. Most of the activities have been tested in the School of Health and Life Sciences at the Universitat Pompeu Fabra, Barcelona, and other Spanish Universities.

Key words: Generic competences. Active learning. Teaching methods.

\section{INTRODUCCIÓN}

El proceso de convergencia hacia el Espacio Europeo de Enseñanza Superior (EEES) ha puesto de relieve la importancia del dominio de competencias transversales durante la formación universitaria $^{1}$. De hecho, señala su desarrollo como uno de los objetivos básicos que deben contemplar las próximas reformas de los planes de estudio tendentes a la armonización universitaria en la Unión Europea. Así, las nuevas orientaciones marcan la necesidad de precisar tanto las competencias específicas de cada titulación como las transversales.

En los últimos años se ha realizado un notable esfuerzo para establecer qué competencias profesionales deberían adquirir los licenciados en ciencias 
de la salud para asegurar que la formación en el período universitario se adapta óptimamente a lo que se requerirá de ellos como graduados. Este objetivo ha sido especialmente importante en las facultades de medicina, donde algunas de ellas, la mayoría de las catalanas ${ }^{2-4}$, han conseguido finalizar el largo camino con el establecimiento de tales competencias en sus centros. Queremos mencionar que la Agencia de la Calidad Universitaria de Cataluña ha definido las competencias que deberían conseguir todos los licenciados en medicina ${ }^{5}$.

El interés de conocerlas es obvio: constituye el elemento básico para juzgar la idoneidad de un plan de estudios, corregirlo cuando no sea así y conocer cuáles son los contenidos básicos que deben evaluarse. Pese a su importancia, su elaboración es compleja y no está exenta de conflictos internos, por lo que muchos planes de estudio aun no disponen de tal documento que consideramos esencial para una actividad docente basada en criterios racionales y no dejada exclusivamente a la intuición de cada profesor.

Tradicionalmente en las titulaciones en ciencias de la salud la atención se ha centrado en las competencias específicas y ha obviado la preocupación por la instauración de ciertas competencias transversales a pesar de ser consideradas como importantes. Por ejemplo, es evidente que la comunicación escrita es una competencia que debe desarrollarse durante los estudios universitarios, pero la mayoría de los estudiantes de ciencias de la salud tienen pocas posibilidades de hacerlo más allá de algunos trabajos esporádicos o de los exámenes en formato de ensayo. En otros estudios, como Periodismo, Traducción e Interpretación o las diversas filologías, esta competencia es considerada, con razón, absolutamente necesaria para ejercer sus profesiones.

Ahora llega el momento del cambio, ya que nadie duda que médicos, diplomados de enfermería, farmacéuticos, odontólogos o veterinarios necesitan adquirir el dominio de muchas competencias transversales. De hecho, tanto el catálogo de competencias para los licenciados en Medicina ${ }^{5}$ como los documentos de las tres facultades de Medicina que han establecido las competencias para sus titulados ${ }^{2-4}$ ya contemplan la asunción de algunas transversales muy relevantes.

Ante la necesidad de cambio comentada y sabiendo de la especial valoración que los empleadores de los profesionales universitarios hacen de las competencias transversales en diversos ámbitos ${ }^{6,7}$, el presente artículo pretende aportar algunos elementos sobre la bondad de su implementación así como diversas posibilidades para fomentarlas en el marco de los procesos docentes de las titulaciones.

\section{LAS COMPETENCIAS TRANSVERSALES: CONCEPTO Y TIPOS}

Por competencia se entiende "pericia, aptitud, idoneidad para hacer algo o intervenir en un asunto importante" ${ }^{\prime} \mathrm{y}$, en educación, es común distinguir entre aquellas que son específicas y las que son transversales o genéricas. Las primeras caracterizan una profesión y la distinguen de otras, por lo que son motivo de especial atención en la formación que lleva a la evaluación sancionadora y al otorgamiento del título académico que reconoce socialmente su adquisición y, con ello, el desempeño de la profesión. En ciencias de la salud, estén formuladas o no de forma explícita, existe un reconocimiento más o menos unánime en lo que debe ser competente un médico, un farmacéutico o un odontólogo. Esta afirmación se sustenta en que los perfiles profesionales de unos y otros son generalmente distinguibles, aunque puedan compartir áreas formativas comunes. En algunas de ellas, no es inhabitual que puedan plantearse conflictos cuando existe un cierto grado de solapamiento. Recuérdese, a modo de ejemplo, el tema de la atención farmacéutica donde se plantea la posibilidad de los farmacéuticos para aconsejar la administración de algunos medicamentos, lo que algunos médicos interpretan como un cierto intrusismo profesional.

Las competencias transversales o genéricas serían aquellas que rebasan los límites de una disciplina para desarrollarse potencialmente en todas ellas. Son habilidades necesarias para ejercer eficazmente cualquier profesión pero no es frecuente que se consideren de forma explícita en una asignatura determinada, al menos en ciencias de la salud. Como ya se ha comentado, en otras áreas, como humanidades o ciencias sociales, algunas de ellas se consideran como indispensables y tienen un espacio curricular asignado, aunque la competencia que recibe este tratamiento varía de una a otra licenciatura.

Por la dificultad en realizar una lista exhaustiva de todas ellas, hemos optado por aceptar la lista propuesta por el proyecto Tuning ${ }^{9}$, posiblemente el que está teniendo más repercusión en el proceso de adaptación a la convergencia europea. La Tabla 1 presenta las 30 competencias transversales de dicho proyecto. 
Creemos que existe una cierta unanimidad al considerar que un buen profesional del ámbito sanitario debe ser capaz de manifestar un desempeño suficiente en la mayoría de las competencias transversales consideradas. No es menos cierto que algunas de ellas tienen un fuerte componente innato y algunos individuos parecen especialmente dotados. En otras ocasiones, las actividades extracurriculares permiten que se alcance un dominio adecuado

Tabla 1. Competencias transversales propuestas en el proyecto "Tunning" sobre la convergencia europea de enseñanza superior.

Instrumentales

Capacidad de análisis y síntesis

Capacidad de organización y planificación

Conocimientos generales básicos

Conocimientos básicos de la profesión

Comunicación oral y escrita

Conocimiento de idiomas

Habilidades en el manejo de un ordenador

Habilidades de gestión de la información (buscar

y analizar información de fuentes diversas)

Resolución de problemas

Toma de decisiones

\section{Interpersonales}

Capacidad crítica y autocrítica

Trabajo en equipo

Habilidades interpersonales

Trabajo en equipo interdisciplinario

Capacidad para comunicarse con expertos de otras áreas

Reconocimiento de la diversidad y multiculturalidad

Habilidad para trabajar en un contexto internacional

Compromiso ético

\author{
Sistémicas \\ Capacidad de aplicar los conocimientos en la práctica \\ Habilidades de investigación \\ Aprendizaje \\ Adaptación a nuevas situaciones \\ Capacidad para generar nuevas ideas (creatividad) \\ Liderazgo \\ Conocimiento de culturas y costumbres de otros países \\ Habilidad para trabajar de forma autónoma \\ Diseño y gestión de proyectos \\ Iniciativa y espíritu emprendedor \\ Motivación por la calidad \\ Motivación por la consecución de objetivos
}

(idiomas, informática), pero no hay duda que el periodo de formación universitaria sería el momento óptimo para plantearse la asunción explícita de muchas de ellas. Hay que crear buenos profesionales desde el principio y no puede dejarse en manos de la improvisación la consecución de este objetivo irrenunciable. Por todo ello, las competencias transversales deben formar parte de los objetivos docentes de los estudios de ciencias de la salud y deben ser formuladas explícitamente entre éstos. Veamos algunas consideraciones al respecto.

Existen pocas dudas que dentro de las competencias transversales instrumentales, las más cognitivas son esenciales para el ejercicio de las diversas profesiones sanitarias. Es evidente que la capacidad analítica y sintética, su aplicación a la resolución de problemas y a la consecuente toma de decisiones forma parte de su actividad diaria. A ella debe añadirse la presencia de un razonamiento crítico que permita que tales profesionales no funcionen como una automática correa de transmisión de órdenes superiores sino como expertos que deciden en función de la aplicación de principios racionales a situaciones específicas. Tales características son las que diferencian a un buen médico, farmacéutico o biólogo del que no lo es y, por tanto, deberían ser estimuladas al máximo durante la formación universitaria.

Pese a la obviedad de lo afirmado en el párrafo anterior, es evidente que en la mayoría de los casos no es así y la situación acaba pervirtiéndose hasta el extremo que los propios estudiantes piden 'recetas' para solucionar problemas antes que aplicar su propio razonamiento para hacerlo. El mayor éxito de los profesores sería conseguir estudiantes díscolos (por indóciles y perturbadores) que desafiaran cualquier conocimiento que fuera transmitido como dogma siguiendo la mejor tradición medieval. Sólo aquellos principios que se basaran en un análisis epistemológico deberían ser aceptados y todo lo demás aceptado transitoriamente hasta que la investigación los compruebe o refute. El modelo docente actual está muy lejos de esta situación y lo cierto es que la aceptación acrítica hace poco por mejorar el estado científico de nuestras disciplinas.

Las competencias transversales instrumentales de tipo lingüístico o tecnológico son asimismo motivo de poca polémica. No hay duda que un diplomado en enfermería o una odontóloga podrán ejercer su profesión de forma adecuada sin conocer una palabra de inglés, francés o alemán. Pero no es menos cierto que la mayoría del conocimiento en 
ciencias de la salud se genera en lenguas ajenas a las nuestras y, por ello, el acceso rápido a él sólo es posible con un grado mínimo de manejo del inglés. Las grandes revistas de ciencias de la salud, las bases de datos internacionales o las reuniones científicas importantes, emplean siempre esta lengua y, por ello, es indispensable para una rápida actualización de conocimientos. Por fortuna, su conocimiento está claramente extendido entre los más jóvenes y en la actualidad es común que la lectura de artículos en inglés o el empleo de programas de simulación en esta lengua no supone un obstáculo insalvable, una vez conocido el vocabulario básico de cada disciplina.

Con las habilidades informáticas la situación es similar, pues aquellos que no dominen los programas de aplicación básicos y no se desenvuelvan mínimamente bien en internet pueden llegar a convertirse en los analfabetos funcionales del siglo XXI. Afortunadamente, el gran esfuerzo realizado en las escuelas primarias y secundarias evitará que esto sea así para las nuevas generaciones, y facilita que estos conocimientos sean aplicados con más facilidad a la búsqueda de información. Pero aquí nos encontramos con otro problema que sí debe constituir una de nuestras actividades docentes: el análisis crítico de la información. Las publicaciones tradicionales y ahora internet permiten el acceso rápido y frecuentemente gratuito a una cantidad ingente de información que tiene una calidad científica muy variable. Es función nuestra contribuir a que nuestros estudiantes aprendan a separar el grano de la paja mediante la aportación de criterios que les ayuden en este sentido y eviten convertirles en profesionales crédulos ante cualquier noticia 0 novedad.

A pesar de que la comunicación oral y escrita sea considerada como una habilidad ya adquirida cuando los estudiantes alcanzan la universidad, lo cierto es que muchos profesores consideramos que con frecuencia no es así. Es más, incluso tras licenciarse numerosos graduados carecen de la capacidad de hacerlo adecuadamente, aunque el análisis de porqué ocurre nos distraería del objetivo de este artículo. Lo que sí es cierto es que debe fomentarse la comunicación escrita mediante la redacción de documentos donde los estudiantes aprendan a presentar de forma lógica y coherente sus puntos de vista sobre un determinado problema. Para ello, el uso de pruebas de ensayo en la evaluación del rendimiento académico no basta aunque debería ser un método para comprobar la bondad de la formación en esta competencia y no un método exclusivamente sancionador de conocimientos teóricos.

De forma similar, la comunicación oral es deficitaria en una amplio número de universitarios, poco habituados a hablar en público y explicar sus puntos de vista con un lenguaje adaptado al registro lingüístico de la situación. La organización de actividades donde puedan aprender a hacerlo es esencial e igualmente debe evitarse la tortura psicológica de exponer los estudiantes a un examen oral para el que nunca han sido preparados.

Las competencias transversales interpersonales constituyen un elemento de importancia excepcional en la formación universitaria de cualquier titulación y, por supuesto, en ciencias de la salud. De entre ellas, destacamos como esencial la capacidad de trabajar en equipo, ya que la situación profesional actual conlleva la realización de proyectos asistenciales o de investigación en colaboración, lo que supone la necesidad de realizar actividades donde se aprenda la dinámica de un grupo de trabajo, la asignación de tareas y el respeto hacia las opiniones del resto de los miembros.

Finalmente, las competencias transversales sistémicas constituyen un conjunto de habilidades que son esenciales para que los estudiantes se conviertan en el futuro en profesionales capaces de los mayores logros. Ello pasa naturalmente por desarrollar la capacidad para aprender autónomamente, pues la formación continuada personal es la única garantía contra la obsolescencia profesional. Junto a ello, debe estimularse, y nunca coartarse, la creatividad, el espíritu emprendedor y la iniciativa. Este requisito es quizá el más fácil de conseguir, pues la juventud lleva inherente la mayoría de aquellas y los profesores solamente deberían encauzar el entusiasmo que nuestros estudiantes tienen. Debe recordarse que, globalmente, los estudios de ciencias de la salud siguen teniendo alumnos muy motivados y capaces (primera opción en la selección de la carrera y notas de acceso a la universidad altas), a pesar de que las perspectivas profesionales no son ahora óptimas. Tenemos pues, si se nos permite el símil gastronómico, las mejores materias primas y sólo debemos aprender a cocinarlas adecuadamente.

\section{CÓMO FOMENTAR LA ASUNCIÓN DE LAS COMPETENCIAS TRANSVERSALES}

Por razones diversas que sería largo de comentar aquí y que son conocidas por la mayoría de los lec- 
tores, los planes de estudio actuales contienen una carga docente muy elevada que dificulta ir más allá de actividades tradicionales como las clases llamadas magistrales, las prácticas (generalmente en grupos demasiado amplios) y algunos seminarios. Existe poco tiempo para considerar el desarrollo de las competencias genéricas, pues muchos profesores sienten que con el tiempo destinado a las competencias específicas no es posible formar adecuadamente a los estudiantes. De nuestras conversaciones hemos deducido que esta creencia está bastante extendida en los ámbitos docentes españoles, pero no es compartida con la misma frecuencia fuera de nuestras fronteras donde los planes de estudios contemplan métodos docentes dirigidos a adquirir y mejorar las competencias transversales al mismo tiempo que las específicas.

Si bien en España las experiencias institucionales en la dirección apuntada no son frecuentes, sí son muchos los profesores que trabajan con gran imaginación y esfuerzo para conseguir que sus estudiantes no sólo aprendan lo que les permita alcanzar las competencias específicas de sus estudios, sino también todas aquellas que harán de ellos unos profesionales aún mejores. Desafortunadamente, pocos de ellos las publican y quedan habitualmente como experiencias locales de escasa difusión.

A partir de nuestra experiencia propia como docentes y en el proyecto educativo de la Facultad de Ciencias de la Salud y de la Vida de la Universidad Pompeu Fabra (UPF) ${ }^{10}$ proponemos al lector una serie de actividades que creemos pueden fomentar el dominio de numerosas competencias transversales durante su formación universitaria.

\section{a) Aprendizaje basado en problemas (ABP)}

Posiblemente el método más adecuado para el desarrollo de competencias transversales seria el aprendizaje basado en problemas (ABP), también conocido como "problem-based learning (PBL)". Es un método y una filosofía docente ampliamente utilizado en la docencia de las ciencias de la salud en el mundo anglosajón y escandinavo. En el año 2000 se calculaba que un $25 \%$ de las facultades de Medicina lo empleaban en los EE.UU. y un 50\% en Canadá $^{11}$. También ha sido recomendado por el General Medical Council británico como el método de elección para mejorar la docencia de la medicina en las facultades del Reino Unido ${ }^{12}$. Sus características, ventajas e inconvenientes pueden ser leídas en las numerosas publicaciones sobre el tema y no insistiremos en esta revisión ${ }^{13}$. Brevemente, se trata de que los estudiantes aprendan por confrontación con problemas reales o simulados que son pertinentes para su formación. Las actividades se realizan en grupos reducidos que trabajan bajo la supervisión de un tutor que actúa como guía del proceso de aprendizaje y que no proporciona las respuestas a los problemas planteados.

El ABP es un método excelente para desarrollar competencias transversales de todo tipo ${ }^{14}$ : instrumentales cognitivas y metodológicas (capacidad de análisis y síntesis, resolución de problemas, toma de decisiones, capacidad de organización y planificación, gestión del tiempo, razonamiento crítico), instrumentales tecnológicas y lingüísticas (búsqueda y análisis crítico de la información, comunicación oral y escrita), interpersonales (trabajo en equipo, habilidades interpersonales, liderazgo, compromiso ético) y sistémicas (capacidad para el aprendizaje autónomo, la adaptación a nuevas situaciones).

$\mathrm{Su}$ puesta en marcha requiere diversos compromisos que implican a los profesores y los órganos responsables de un centro universitario, aunque existen experiencias de inferior nivel que también han funcionado. De entre las experiencias llevadas a cabo institucionalmente, destacamos las de la Facultad de Medicina de la Universidad de CastillaLa Mancha ${ }^{15}$ y de la Escuela de Enfermería del Hospital Vall d'Hebron de la Universitat Autónoma de Barcelona ${ }^{16}$. En ambos centros, se aplica el modelo de aprendizaje basado en problemas (ABP) en todo el ciclo de estudios, una apuesta difícil que merece nuestro respeto y admiración. Ambas han graduado su primera promoción de estudiantes formados con el ABP.

Queremos recordar que la implantación institucional del ABP puede crear fuertes resistencias, especialmente cuando es el utilizado como único método de aprendizaje o de forma predominante. Ante este hecho, otra posibilidad es su empleo como método docente coexistente con las clases magistrales, lo que también permite alcanzar muchos de los objetivos propuestos con un número de resistencias menor $^{17}$. En la Facultad de Ciencias de la Salud y de la Vida de la UPF, se está utilizando el ABP en quinto curso de la licenciatura en Biología desde hace tres años, ocupando alrededor del $30 \%$ de los horas docentes del único trimestre presencial (durante los dos trimestres restantes los estudiantes realizan practicas profesionalizadas en diversas empresas o instituciones $)^{18}$. 
Sus resultados positivos nos han llevado a extender la experiencia a todos los cursos. En el curso académico 2004-05 hemos estamos inmersos en una experiencia piloto, impulsada por las autoridades educativas catalanas, para adaptar nuestros estudios a las nuevas orientaciones de la convergencia europea. Así, la principal innovación de la experiencia piloto ha consistido en la iniciación de actividades de ABP como parte del aprendizaje de todas y cada una de las asignaturas. Este tipo de enseñanza que se plantea de forma interdisciplinaria (los problemas son de diversas asignaturas) ocupa aproximadamente un $20 \%$ del horario lectivo ${ }^{19}$.

Así mismo y al margen de proyectos institucionales, desde hace años también existen otras experiencias de $\mathrm{ABP}$ en algunas asignaturas de la licenciatura de Medicina, como en Anestesiología y Reanimación en la Universitat Autónoma de Barcelona $^{20}$.

\section{b) Simposios y minicongresos}

Los simposios y minicongresos son también buenas oportunidades de estimular a los estudiantes en la senda de su propio aprendizaje. La organización de pequeñas reuniones científicas dentro de la facultad o escuela donde los estudiantes presentan los resultados de un trabajo de revisión realizado en grupo durante unos meses puede convertirse en una magnífica ocasión para desarrollar múltiples competencias transversales. En este sentido, en nuestro centro se realizan diversas actividades de este tipo como serían las presentaciones a través de carteles que realizan grupos de un número reducido de estudiantes en dos asignaturas de nuestros estudios (Fisiopatología y Evolución).

Dentro de este apartado nos gustaría comentar una jornada de divulgación científica que se realiza anualmente en nuestra Facultad dentro del proyecto docente de la asignatura de Microbiología. En dicha jornada los estudiantes de tercer curso que la cursan presentan sus trabajos de forma oral en el auditorio de la Facultad. Los temas escogidos son de actualidad y en las últimas jornadas se centraron en bioterrorrismo y enfermedades infecciosas emergentes. Las sesiones son abiertas a profesores, alumnos de todos los cursos y familiares. Suponen un acontecimiento para los estudiantes y los resultados son muy satisfactorios. Esta es una opción al alcance de cualquier centro que se ponga manos a la obra en su organización.
Los minicongresos de estudiantes suponen una loable iniciativa que se desarrolla en algunas universidades. Más allá de las reuniones organizadas por las asociaciones de estudiantes, los minicongresos son actividades que pueden organizarse desde la propia facultad o escuela y, en ocasiones, desde los departamentos. Cabe destacar al respecto la larga experiencia de los minicongresos de farmacología organizados por el Departamento de Farmacología y de Terapéutica de la Universidad Autónoma de Madrid ${ }^{21}$. Desde hace varios años, este Departamento organiza una reunión científica, que primero fue local y ahora ya de ámbito estatal, en la que los estudiantes presentan sus trabajos originales o de revisión sobre los temas de su interés, siempre en el amplio ámbito de la farmacología. Como en cualquier otro congreso, existen también conferencias plenarias realizadas por profesionales de prestigio. Los organizadores han contado con soporte institucional y de algunas empresas privadas para su realización.

No hace falta destacar qué competencias transversales son desarrolladas con estos dos tipos de actividades y hay pocas dudas de que contribuyen notablemente a la formación de los estudiantes que las siguen, ya que suponen un primer contacto para aprender cómo comunicar en ciencia, una habilidad que les será sin duda muy útil para su futuro profesional.

\section{c) Uso del cine comercial}

La utilización de películas comerciales en el ámbito educativo es una experiencia de notable interés también en las ciencias de la salud ${ }^{22}$. Consiste en la proyección de películas que se exhiben en las salas comerciales o en la televisión con objetivos docentes. Es frecuente que vayan seguidas de un debate sobre los aspectos más relevantes que se muestran en ellas pero existen otras posibilidades. Su interés viene refrendado por el aumento progresivo de publicaciones sobre el tema ${ }^{22-25}$ y por la publicación de una nueva revista ${ }^{26}$ destinada a recoger y difundir experiencias en este ámbito.

En el campo de la medicina, por ejemplo, existen diversas experiencias sobre el uso de películas y autores españoles han publicado artículos sobre su empleo en la docencia de la farmacología $a^{27}$ y de la microbiología ${ }^{28}$. También tenemos conocimiento en su uso en el aprendizaje de psicología médica ${ }^{29}$, psiquiatría $^{30}$, historia de la medicina ${ }^{31}$, ética ${ }^{32} 0$ derecho ${ }^{33}$. 
Esta estrategia docente puede permitir ilustrar situaciones complejas de difícil comprensión para los estudiantes de ciencias de la salud en sus primeros años (compromisos éticos, relación médicopaciente, límites de la investigación). Las películas disponen de muchos de los elementos que inducen a la reflexión: excelentes medios técnicos, buenos argumentos, magníficos actores, verosimilitud y capacidad de seducción. Si estas actividades se organizan adecuadamente, los estudiantes pueden aprender a analizar críticamente un problema, razonar lo expuesto desde diversos puntos de vista, expresar oralmente y por escrito sus puntos de vista $^{34}$.

En nuestra Facultad, el cine comercial es utilizado por diferentes asignaturas a lo largo de los estudios de Biología (Introducción a la Biología, Psicobiología, Microbiología, Psicología, Bioética) con objetivos diversos (discusión en seminario, debate sobre algún tema relevante, reflexión sobre aspectos éticos, ilustración del desarrollo de alguna enfermedad o base para evaluaciones formativas). Pero queremos destacar una actividad, que bautizamos como Biocinema, donde los estudiantes de quinto y último curso, que siguen tres itinerarios formativos diferentes, se reúnen periódicamente para ver una película sobre temas de interés común, discutirla posteriormente y realizar un pequeño escrito sobre los elementos que ellos consideren como más relevantes ${ }^{35}$.

Contrariamente a otras actividades comentadas en este artículo, el uso del cine comercial con objetivos docentes es una actividad barata, fácil, versátil y útil en múltiples contextos. Con una evaluación adecuada puede ser un método valioso para estimular la reflexión de los estudiantes y mejorar incluso sus conocimientos y habilidades específicas en muchos temas.

\section{d) Trabajos de investigación}

Los trabajos de investigación han sido empleados ampliamente en otras licenciaturas pero menos en algunos de los estudios de ciencias de la salud. Supone la realización de un trabajo original sobre algún tema acordado entre alumnos y profesores y no un mero trabajo de revisión. No siempre es necesario que sea de laboratorio, porque los que incluyen encuestas, revisiones sistemáticas o el uso de instrumentos psicológicos también son aceptables. Los trabajos de investigación pretenden desarrollar en los alumnos su interés por el método científico, la búsqueda de información, el trabajo en grupo o la gestión del tiempo, entre otras competencias transversales.

Querríamos comentar la experiencia de la Facultad de Medicina del País Vasco, donde los estudiantes de farmacología pueden realizar un trabajo de investigación en lugar de las prácticas tradicionales de la asignatura ${ }^{36}$. Los autores realizaron un estudio aleatorizado y comparativo entre los dos grupos y mostraron un elevado grado de satisfacción y de aprovechamiento en el primer grupo ${ }^{37}$. Esta experiencia debe tenerse en cuenta en aquellos centros en los que el número de estudiantes no constituye óbice para su implantación y en los que exista un grupo mínimo de profesores que decidan implicarse. Existen también otras experiencias en el ámbito de la docencia clínica ${ }^{38}$.

En nuestra Facultad contemplamos dos actividades para fomentar el interés por la investigación y el fomento de las competencias transversales asociadas a su desarrollo. Por un lado estimulamos las estancias de investigación durante el verano en algún grupo investigador tanto vinculado a nuestro departamento o a grupos cercanos como a instituciones nacionales o extranjeras. Disponemos de tres programas reconocidos, uno de ellos de la Asociación de Estudiantes de Ciencias de la Salud, donde los alumnos después de realizar su estancia de investigación pueden obtener créditos de libre elección al presentar un informe del tutor y una memoria con unos requisitos concretos. Esta experiencia, que es ampliamente aceptada, permite a los estudiantes realizar trabajos interesantes que en bastantes casos han fructificado en comunicaciones a congresos e incluso en publicaciones internacionales.

Por otro lado, los estudiantes del último curso realizan un trabajo de investigación durante sus prácticas profesionalizadas que han de presentar en una sesión de carteles en la jornada de graduación, donde dicha actividad se realiza junto a un acto académico formal ante la asistencia de los miembros de la comunidad universitaria del centro (estudiantes, profesores y profesionales de administración y servicios) y de familiares y amigos. Hasta el momento, el resultado de dicha actividad ha sido claramente satisfactorio.

\section{e) Visitas a centros del patrimonio histórico-sanitario}

Las visitas a centros del patrimonio históricosanitario pueden también emplearse para comple- 
tar y mejorar la formación de los estudiantes en diversas áreas. España dispone de un amplio número de instituciones susceptibles de interés para los estudiantes de ciencias de la salud como se recoge en dos obras publicadas recientemente, la primera dedicada a la ciudad de Barcelona ${ }^{39}$ y la segunda a toda España ${ }^{40}$. Estas instituciones incluyen museos, hospitales, empresas y otras instalaciones que pueden tener un notable interés docente.

Cualquier lector podría dar múltiples ejemplos pero recordaremos aquí algunos de Cataluña que conocemos bien. Así, tiene notable interés el Museo de la Farmacia ubicado en los Laboratorios AlcónCusí (El Masnou) para observar la evolución de las farmacias desde la época barroca hasta la actualidad. Para conocer el devenir de la asistencia hospitalaria, una visita combinada a la Biblioteca de Cataluña, actual sede del medieval Hospital de la Santa Creu fundado en 1401, con el modernista Hospital de la Santa Creu i Sant Pau, ambos en Barcelona, ayuda a comprender cómo los avances científicos se plasmaron en los cambios arquitectónicos. Los estudiantes comprenderán mejor la asistencia psiquiátrica si se les lleva a un hospital de la época previa a la psicofarmacología, como el impresionante Institut Pere Mata (Reus) diseñado por Doménech i Muntaner y excelente muestra de la arquitectura modernista. También pueden entender el inicio de la investigación biomédica catalana con la visita a la sede de l'Institut d'Estudis Catalans, donde se encuentra la Societat Catalana de Biologia, que tuvo un papel decisivo en la génesis de aquella. En nuestra Facultad hemos utilizado estas visitas dentro de la asignatura de Historia de la Biología y de la Medicina ${ }^{41}$, pero tenemos pocas dudas de su interés para otras materias como Salud Pública, Psiquiatría, Farmacología o Pediatría, por poner algunos ejemplos.

\section{f) El uso de Portafolios}

Finalmente queremos referirnos al uso de portafolios en el fomento de las competencias transversales. Los portafolios son unos instrumentos que tienen dos grandes finalidades, por un lado permiten la reflexión sobre el desarrollo de una competencia con la intención de mejorarla y por otro permiten acreditar el grado de asunción de dicha competencia. Así, tienen un valor formativo (reflexión para la mejora) y pueden tener un valor acreditativo (documentar algún logro). La utilización de portafolios por los estudiantes está siendo recomendado ya que supone una importante contribución al autoaprendizaje basado en la propia reflexión del alumno ${ }^{42}$.

Desde sus inicios, uno de los objetivos institucionales de nuestro centro ha pretendido que nuestros estudiantes, al acabar su licenciatura, dominen cuatro competencias transversales que consideramos prioritarias: hablar en público delante de un auditorio, escribir correctamente, recoger información a través de las nuevas tecnologías y trabajar en equipo. Si bien estas competencias se han venido fomentando en el seno de las diversas asignaturas a través de actividades propias, hasta hace relativamente poco no se han explicitado como objetivo institucional. Desde hace dos años, los estudiantes del último curso pueden realizar un portafolio sobre las cuatro competencias señaladas que tiene contingencia sobre la nota de las prácticas profesionalizadas.

Así mismo, aprovechando la experiencia piloto sobre la adaptación europea en la que estamos inmersos y que hemos comentado anteriormente ${ }^{19}$, hemos introducido esta actividad desde el primer curso. Pretendemos que nuestros estudiantes reflexionen sobre las cuatro competencias, detecten sus puntos fuertes y los débiles, hagan propuestas de mejora y ejerciten dichas propuestas. Así mismo queremos que sean capaces de seleccionar la documentación adecuada para acreditar el aprendizaje realizado a través del tiempo mientras duran sus estudios en las competencias citadas. Para realizar dicha actividad cuentan con un tutor de referencia y con una guía detallada que ha sido elaborada por la oficina educativa del centro ${ }^{43}$.

\section{CONCLUSIONES}

La convergencia europea de enseñanza superior, cuyas directrices aparecen en la Declaración de Bolonia, tiene entre sus principios facilitar el desarrollo de las competencias transversales y el autoaprendizaje. Creemos que se precisa de actuaciones específicas para poder conseguirlas y que la imaginación es necesaria para diseñar algunas de ellas. En el presente artículo hemos recogido, sin ánimo de exhaustividad algunas iniciativas concretas de las que tenemos experiencia directa por haberlas implantado con resultados positivos. No obstante, no hay duda que cada profesor y/o institución precisa de la adaptación de las propuestas o de otras para poder alcanzar los objetivos propuestos que tanto valoran los futuros empleadores. 


\section{BIBLIOGRAFÍA}

1. Hernanz ML, Rosselló G, Canela E, Carbonell J, Estelrich P, Grifoll J, Lorenzo U, Naik AR, Úbeda, E. Marc general per a la integració europea. Barcelona: Agència per a la Qualitat del Sistema Universitari a Catalunya, 2004.

2. Facultad de Medicina de la Universitat de Barcelona. Competències que han d'adquirir els estudiants de Medicina durant els estudis de pregrau a la Facultat de Medicina de la Universitat de Barcelona. Barcelona: Universitat de Barcelona, 2003.

3. Facultat de Medicina de la Universitat de Lleida. Competències professionals a assolir durant el període de formació de pregrau. Lleida: Universitat de Lleida, 2004.

4. Facultat de Medicina i Ciències de la Salut de Reus. Universitat Rovira i Virgili. Competències professionals dels llicenciat en Medicina (a assolir durant el pregrau). Catàleg de competències del llicenciat, FMCS-URV, 2004. http://www. upf.edu/bolonya/butlletins/2004/ setemb2/compet.pdf Consultado el 11 de Febrero de 2005.

5. Prat J, Carreras J, Branda L, Miralles R, Fenoll MR, Rodríguez S, Grifoll J. Competències professionals bàsiques comunes dels llicenciats en Medicina formats a les Universitats de Catalunya. Barcelona: Agència per a la Qualitat del Sistema Universitari a Catalunya, 2004.

6. Hughes I, Hollingsworth, Jones SJ, Markham T. Knowledge and skills needs of pharmacology graduates in first employment: how do pharmacology courses measure up? TiPS 1997; 18:111-116.

7. Ayats Salt JC, Samora Suárez P, Desantes Fernández R. Los titulados de la Universidad Politécnica de Valencia y los empleadores. Valencia: Universidad Politécnica de Valencia, 2004.

8. Real Academia Española. Diccionario de la Lengua Española. Vol 1. 22a ed. Madrid: Espasa, 2001; p. 604.

9. González J, Wagenaar R. Tunning Educational Structures in Europe. Informe final. Deusto: Universidad de Deusto, 2003.

10. Pérez, J. L'impuls al debat pedagògic: Projecte educatiu de la Facultat de Ciències de la Salut i de la Vida de la Universitat Pompeu Fabra. En: AQU. La implantació de les millores. Barcelona: Agència per a la Qualitat del sistema Universitari a Catalunya, 2001.

11. Spencer JA, Jordan RK. Learner centered approaches in medical education. Brit Med J. 1999; 318:12801283.

12. General Medical Council. Tomorow's doctors. Recommendations on undergraduate medical education. Londres: General Medical Council, 2002.
13. Wood DF. Problem based learning. Brit Med J. 2003; 326:328-330.

14. Walton HJ, Matthews MB. Essentials of problembased learning. Med Educ 1989; 23:542-558.

15. Alfonso Roca MT, Sáez Méndez L, Serrano Casarrubio P. Changing Spanish medical studies: our experience. Educ Med 2004; 7(3): 268.

16. Quintanilla M, Bernaus E, Guillamet A, Fernández A. Learning to solve problems in clinical practice. Educ Med 2004; 7(3): 104.

17. Baños JE. El aprendizaje basado en problemas en los planes de estudio tradicionales: ¿una alternativa posible? Educ Med 2001; 4:4-12.

18. Baños JE, Aramburu J, Bosch F, Pérez J, Sentí M. Enhancing the pre-graduation contact with the real world: the experience of professional tracks in human biology students at the Universitat Pompeu Fabra of Barcelona. Educ Med 2004; 7(3): 48.

19. Pérez J, Baños JE. Acceptant un gran repte. AQU Notícies 2004; octubre.

20. García-Guasch R, Lucas M, Moret E. Problem-based learning in undergraduate teaching of anesthesiology. Educ Med 2004; 7(3) 105.

21. Sánchez-Ferrer C F. Minicongreso de farmacología: 22 años de una experiencia docente en el Departamento de Farmacología de la UAM. Educ Med 1999; 2:195.

22. García Sánchez JE, Trujillano Martín I, García Sánchez E. Medicina y cine ¿Por qué?. J Med Mov 2005; 1:1-2.

23. González Blasco P. Literature and movies for medical students. Fam Med 2001; 33:426-428.

24. Lee YM, Ahn DS. Medical themed film and literature course for premedical students. Med Teacher 2004; 26:534-539.

25. Alexander M. The Doctor: A seminal video for cinema education. Fam Med 2002; 34: 92-94.

26. http://www3.usal.es/ revistamedicinacine/ Consultada el 11 de Febrero de 2005.

27. Farré M, Bosch F, Roset PN, Baños JE. Putting clinical pharmacology in context: the use of popular movies. J Clin Pharmacol 2004; 44:30-36.

28. García Sánchez JE. Fresnadillo Martínez MJ, García Sánchez E. El cine en la docencia de las enfermedades infecciosas y la microbiología clínica. Enferm Infec Microbiol Clin 2002; 20:403-406.

29. Bosch, F, Martínez, A, Pérez, J. Cinema comercial i educació mèdica. Dits i Fets, 1997; 1(4): 3.

30. Iruela LM. Cine y psiquiatría. Arch Psiquiat 2004; 67:73-74.

31. Cañizo Fernández-Roldán A. El experimento Tuskagee. Estudio de la evolución de la sífilis en pacientes negros no tratados. J Med Mov 2005; 1:12-16 
32. Patricio MF, Lacerda AP, Sá P, Gomes-Pedro J. “To be a doctor": teaching-learning attitudes using commercial films for discussion on ethical dilemmas. Educ Med 2003; 6 (3):48.

33. Crawford C. Law enforcement and popular movies: Hollywood as a teaching tool in the classroom journal of criminal justice and popular culture 1999; 6: 46-57.

34. Baños JE, Bosch F. Las películas comerciales en la docencia de la medicina: más cine, por favor. Rev And Patol Dig, 2005; 28:10-13.

35. Baños JE, Aramburu J, Sentí M. Biocinema: the experience of using popular movies with students of Biology. J. Med Mov, sometido a publicación, 2005;1:42-46.

36. Tamayo G, Santibáñez M, Meana JJ. De la enseñanza tradicional al aprendizaje basado en el desarrollo de un proyecto de investigación. Una experiencia de transición en Farma-cología. Educ Med 2000; 3:25-33.

37. Santibáñez M, Tamayo G, Meana JJ. Percepción de los estudiantes sobre un modelo experimental de actividad práctica en farmacología. Un análisi controlado, aleatorizado y bajo condiciones de enmascaramiento. Edu Med 2000; 3:69-76.

38. Chung C, Gutiérrez M, Gómez R. Promoción de la formación médica continuada de los estudiantes de medicina mediante resolución de un tema de investigación. Ed Med 2004; 6:60.

39. Durán X, Piqueras M. Passejades per la Barcelona científica. Barcelona: Ajuntament de Barcelona, 2002.

40. Maldonado JL, García González A. La España de la técnica y de la ciencia. Madrid: Acento, 2002.

41. Baños JE, Pérez J, Presas A. El patrimoni històric i mèdic de Catalunya com a eina docent en les Ciències de la Salut. Ann Med 2004; 87:41-5.

42. Mateo J, Arboix E, Barà J, Ferrer F, Font J, Monreal P, Pérez J, Sangrà A. Marc general per a l'avaluació dels aprenentatges dels estudiants. Bercelona: Agència per a la Qualitat del Sistema Universitari a Catalunya, 2003.

43. Valero M, Pérez, J. Guia del Portafoli per als estudiants de primer. Barcelona: Facultat de Ciències de la Salut i de la Vida. Universitat Pompeu Fabra, 2005. 